\title{
Modified “Current” Statisical Model Filtering Algorithm for Carrier Acceleration Calculation
}

\author{
Zhaolong Zhang*, Yuegang Wang, Honglei Teng, Le Wang \\ Xi'an High Tech Research Institute, 2 Tongxin Road, Baqiao District, Xi'an 710025, China \\ ${ }^{*}$ Corresponding author
}

\begin{abstract}
Carrier acceleration is an important factor that influences the solution of the gravity anomaly in airborne gravimetry. The development of the airborne gravimetry has put forward a higher requirement to carrier acceleration. Considering the complex case of high-flying carrier in airborne gravimetry, in order to improve the solution accuracy of carrier acceleration, this paper adopt the Kalman filter method based on "Current" Statistical Model to solve the problem. For the characteristics of carrier acceleration. The fuzzy membership function and the Interacting Multiple Model algorithm are used to adjust the acceleration limits and motor frequencies of the "Current" Statistical Model. An improved "Current" Statistical Model algorithm is proposed to solve the carrier acceleration of airborne gravimetry, and then the filtering results are smoothed by the RTS smoothing. Finally, the proposed algorithm is validated by simulation experiments, the simulation results show that the proposed method is superior than the existing method position differential method in solving carrier acceleration, and the solution accuracy is improved greatly.
\end{abstract}

Keywords-airborne gravimetry; carrier acceleration; interacting multiple model; fuzzy adaptive ; "current" statistical model

\section{INTRODUCTION}

In the airborne gravimetry, it is necessary to remove the carrier acceleration from the specific force measured by the gravimeter in order to obtain the effective value of the Earth's gravity anomaly[1-3]. In recent years, the problem of solution of the carrier acceleration correction is the hotspot in the field of airborne gravimetry. At present, the widely used methods of carrier acceleration calculation are digital difference and FIR low-pass filtering, including position differential method, Doppler frequency shift method and phase timing difference method[4-6]. However, since the numerical difference process amplifies the high-frequency noise of the positioning error, the accuracy of the acceleration calculation is affected, too. In order to further improve the accuracy of airborne gravimetry, we need to choose a more effective solution method.

To avoid the amplification of noise, The acceleration signal and noise can be reasonably estimated by constructing a model to distinguish them, and to resolve the aliasing as much as possible. Kalman filter is a time-domain filtering method which is based on model, and has been widely used in airborne gravimetry in recent years. For carrier acceleration calculations, the important thing is to establish a model that conforms to the actual motion of the carrier. The "Current" Statistical Model (CS) uses the modified Rayleigh Distribution to describe the "current" acceleration of the moving target, which can reflect the acceleration change of the target more effectively. At present, the model has been successfully applied in target tracking, track forecasting and other fields[7-8].

In this paper, the "Current" Statistical Model is applied to the carrier acceleration calculation of airborne gravimetry. Aiming at the problem that the acceleration parameters of CS model can not be adjusted adaptively, the fuzzy membership function is used to fuzzy adaptive correct the extreme value of the acceleration, also the Interacting Multiple Model (IMM) is introduced into the CS model. Finally, The RTS smoothing is used to pcorrect the filter results. The feasibility and effectiveness of the algorithm for carrier acceleration calculations of airborne gravimetry are verified by simulation experiments.

\section{KALMAN FILTER OF "CURRENT" STATISTICAL MODEL}

Discrete state equation and observation equation of the "Current" Statistical Model can be described as:

$$
\begin{aligned}
& X_{k+1}=\Phi_{k} X_{k}+U_{k} \bar{a}_{k}+W_{k}, W_{k} \square N\left(0, Q_{k}\right) \\
& Z_{k}=H_{k} X_{k}+V_{k}, V_{k} \square N\left(0, R_{k}\right)
\end{aligned}
$$

and,

$$
\begin{aligned}
& \Phi_{k}=\left[\begin{array}{ccc}
1 & T & \left(\alpha T-1+e^{-\alpha T}\right) / \alpha^{2} \\
0 & 1 & \left(1-e^{-\alpha T}\right) / \alpha \\
0 & 0 & e^{-\alpha T}
\end{array}\right] \quad U_{k}=\left[\begin{array}{c}
\left(-\alpha T+\alpha^{2} T^{2} / 2+\left(1-e^{-\alpha T}\right)\right) / \alpha^{2} \\
\left(\alpha T-\left(1-e^{-\alpha T}\right)\right) / \alpha \\
1-e^{-\alpha T}
\end{array}\right] \\
& X_{k}=\left[\begin{array}{lll}
x & \dot{x} & \ddot{x}
\end{array}\right]^{T} H_{k}=\left[\begin{array}{lll}
1 & 0 & 0
\end{array}\right]
\end{aligned}
$$

where, $\bar{a}_{\mathrm{k}}$ is average value of the "current" acceleration, which is equal to the estimated value of the previous time. $\mathrm{T}$ is the sampling period and $\alpha$ means maneuvering frequency. $W_{k}$ is white Gaussian noise and its variance is $Q_{k}=2 \alpha \sigma_{a}^{2} Q_{0}$, $Q_{0}$ is constant matrix:

$$
Q_{0}=\left[\begin{array}{lll}
q_{11} & q_{12} & q_{13} \\
q_{21} & q_{22} & q_{23} \\
q_{31} & q_{32} & q_{33}
\end{array}\right]
$$

$q_{11} \square q_{33}$ can be expressed by the following formulas: 


$$
\begin{aligned}
& q_{11}=\left(1-e^{-2 \alpha T}+2 \alpha T+2 \alpha^{3} T^{3} / 3-2 \alpha^{2} T^{2}-4 \alpha T e^{-\alpha T}\right) / 2 \alpha^{5} \\
& q_{12}=q_{21}=\left(e^{-2 \alpha T}+1-2 e^{-\alpha T}+2 \alpha T e^{-\alpha T}-2 \alpha T+\alpha^{2} T^{2}\right) / 2 \alpha^{4} \\
& q_{13}=q_{31}=\left(1-e^{-2 \alpha T}-2 \alpha T e^{-\alpha T}\right) / 2 \alpha^{3} \\
& q_{22}=\left(4 e^{-\alpha T}-3-e^{-2 \alpha T}+2 \alpha T\right) / 2 \alpha^{3} \\
& q_{23}=q_{32}=\left(e^{-2 \alpha T}+1-2 e^{-\alpha T}\right) / 2 \alpha^{2} \\
& q_{33}=\left(1-e^{-2 \alpha T}\right) / 2 \alpha
\end{aligned}
$$

Kalman filter of "Current" Statistical Model can be expressed as:

$$
\begin{aligned}
& \hat{X}_{k / k-1}=\Phi_{k, k-1} \hat{X}_{k-1}+U \bar{a}_{k} \\
& P_{k / k-1}=\Phi_{k / k-1} P_{k-1} \Phi_{k / k-1}^{T}+Q_{k-1} \\
& K_{k}=P_{k / k-1} H_{k}^{T}\left(H_{k} P_{k / k-1} H_{k}^{T}+R_{k}\right)^{-1} \\
& \hat{X}_{k}=\hat{X}_{k / k-1}+K_{k}\left(Z_{k}-H_{k} \hat{X}_{k / k-1}\right) \\
& P_{k}=\left(I-K_{k} H_{k}\right) P_{k / k-1}
\end{aligned}
$$

The variance of "Current" acceleration $\sigma_{a}^{2}$ can be expressed as:

$$
\sigma_{a}^{2}=\left\{\begin{array}{l}
\frac{4-\pi}{\pi}\left[a_{\max }-\bar{a}\right]^{2}, \bar{a} \geq 0 \\
\frac{4-\pi}{\pi}\left[a_{-\max }-\bar{a}\right]^{2}, \bar{a}<0
\end{array}\right.
$$

where, $a_{\max }$ is maximum acceleration and $a_{-\max }$ is minimal acceleration. Analysis the formula (5), when the appropriate value is selected, the acceleration of the carrier can be calculated more accurately within a certain range. However, in the actual airborne gravimetry, the "current" acceleration variance needs to be adjusted adaptively to obtain higher precision results due to the influence of the atmospheric turbulence and other factors.

\section{IMPROVEMENT OF "CURRENT" STATISTICAL MODEL}

The extreme value of acceleration of the CS model is priori and remains constant during the filtering process, which reduces the estimation precision of the acceleration of Kalman filter. In order to realize the adaptive adjustment of the acceleration extreme value in the filtering process, the fuzzy membership function can be used to fuzzy adaptive correct the extreme value of the acceleration, so it is the fuzzy adaptive "Current" Statistical Model.

Using the bell function as fuzzy membership function, the expression is:

$$
M=\left\{\begin{array}{c}
e^{-\frac{\left(a-a_{\max }\right)^{2}}{2 \sigma^{2}}}, a \geq 0 \\
e^{-\frac{\left(a-a_{-\max }\right)^{2}}{2 \sigma^{2}}}, a<0
\end{array}\right.
$$

Obviously, the formula (6) obeys the normal distribution with $a_{\max }$ or $a_{-\max }$ as the mean and $\sigma^{2}$ as the variance, and $\sigma^{2}$ controls its shape.

The extreme value of acceleration of the adjusted CS model adaptively changes with the function $M$, can be expressed as:

$$
\left\{\begin{array}{cl}
a_{\text {max_new }}=M a_{\max }, & a \geq 0 \\
a_{- \text {max_new }}=M a_{-\max }, & a<0
\end{array}\right.
$$

The size of the maneuvering frequency of the CS model affects the estimation accuracy of the carrier's motion with different properties. The smaller the value of $\alpha$ is, the higher the estimation accuracy of the weak motor carrier. The higher the value of $\alpha$ is, the higher the estimation accuracy of the strong motor carrier. In general, the maneuvering frequency of a single model is fixed and it is difficult to take into account the different maneuvering characteristics performed by the same motion carrier. Therefore, CS models with a number of different maneuvering frequencies are used to describe the carrier motion, and the interaction between each model is realized by using the Interacting Multiple Model (IMM) algorithm, Make full use of the models with different maneuvering frequencies to estimate the motion of the carrier.

The basic principle of IMM algorithm is to build a model set with multiple models, $M=\left\{m_{i} \mid i=1,2, \cdots, m\right\}$, For $\mathrm{m}$ models, the initial state $x_{i}(0)=\hat{x}_{i}(0)$ and the initial model probability $u_{i}(0)=P\left(m_{i}(0) \mid z(0)\right)$ are input. In the process of interaction, through this three procedures: interaction, filtering and fusion, the model probability of each filter cycle is calculated and updated, and update the model set with the new model probability to realize the "Soft Switch" between the models, so as to achieve the automatic adjustment of the filter bandwidth. The specific interaction process is described in the literature[9].

Through the IMM process based on CS model with multiple different maneuvering frequencies, to adjust of the CS model using the model probability which has been calculated automatically, thus realize the further adjustment of the variance of the model acceleration. Achieving the adjustment of maneuvering frequency and expanding the application scope at the same time.

The corrected variance of the acceleration is expressed as follows:

$$
\sigma_{a}^{2}=\left\{\begin{array}{l}
\frac{4-\pi}{\pi}\left[a_{\text {max }_{-} \text {new }} u_{j}(k)-\bar{a}\right]^{2}, \bar{a} \geq 0 \\
\frac{4-\pi}{\pi}\left[a_{- \text {max }_{\text {new }}} u_{j}(k)-\bar{a}\right]^{2}, \bar{a}<0
\end{array}\right.
$$

Equation (8) is the core formula of the fuzzy adaptive "Current" Statistical Model algorithm (IMM-FACS)based on IMM. The acceleration of the carrier can be calculated by 
filtering using the Kalman filtering process expressed as the formula (4) with the variance of the acceleration calculated by the formula (8).

\section{RTS SMOOTHING}

The calculation of the carrier acceleration in airborne gravimetry is the post-processing for the measured data. Thus, the RTS (Rauch Tung Striebel) is used to smooth the data, RTS Smoothing is used to estimate the state variables at each time of a interval using the whole measurement datas in the certain time interval. The accuracy of the estimation of the state is higher than that of the Kalman filter which only use the current time.

\section{V.SiMULATION VERIFICATION}

Laboratory gravity measurement and navigation application experimental system using the scalar gravity method, the vertical component of carrier acceleration is used to calculate the gravity anomalies, the vertical acceleration determines the accuracy and resolution of gravity anomaly. therefore, simulation test mainly studies the vertical component of the carrier acceleration. In order to verify the validity of the proposed method in the application of airborne gravimetry, a simulation signal of simulate DGPS high precision positioning vertical directional position sequence is generated by using laboratory computers in Matlab environment. Using the positional differential method which has been widely used and the method carried out in this paper to calculate the acceleration. Then compare and analyze he results of the two methods. The experimental schemes are as follows:

Option 1: Use the position differential method to calculate the acceleration. The digital difference method uses the second-order central difference and the Hamming window method FIR low-pass filter, the cut-off frequency is $0.01 \mathrm{~Hz}$, the filter length is 300 .

Option 2: Use the IMM-FACS with RTS method, IMM uses the CS model with three different maneuver frequencies, set as $1 / 10,1 / 20$, and $1 / 60$, respectively. The algorithm parameters are set to: $a_{\max }=10 \mathrm{~m} / \mathrm{s}, a_{-\max }=-10 \mathrm{~m} / \mathrm{s}$, $T=1 s$, the initial probability matrix is set to:

$$
\mathrm{P}=\left[\begin{array}{lll}
0.99 & 0.01 & 0.02 \\
0.01 & 0.99 & 0.01 \\
0.02 & 0.01 & 0.99
\end{array}\right]
$$

The result of the IMM-FACS RTS method also uses the same FIR low-pass filter to filter, and then comparing with the result of option 1.

Figures I and II show the results of carrier acceleration of option 1 and 2 without low-pass filtering. It can be seen from the figure that the error of the position differential method is large, which is caused by the amplification of the high frequency noise in the second-order difference process. The error of the method put forward in this paper is small, which is due to the reasonable description of the estimation of the acceleration, which can avoid enlarging the noise.

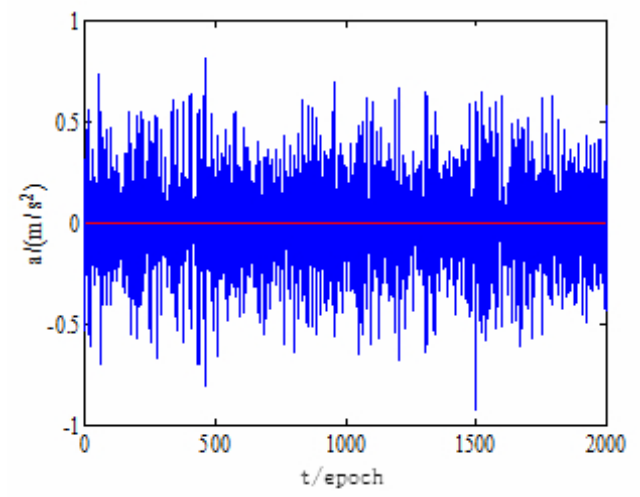

FIGURE I. THE RESULTS OF POSITION DIFFERENTIAL METHOD

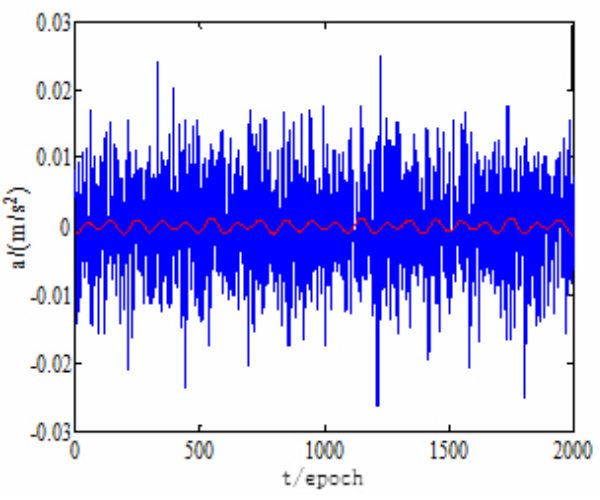

FIGURE II. THE RESULTS IMM-FACS+RTS

And then deal with the results of this two methods with FIR low-pass filter, comparing the the calculated acceleration results after filtering with theoretical value, as shown in Figure III: 


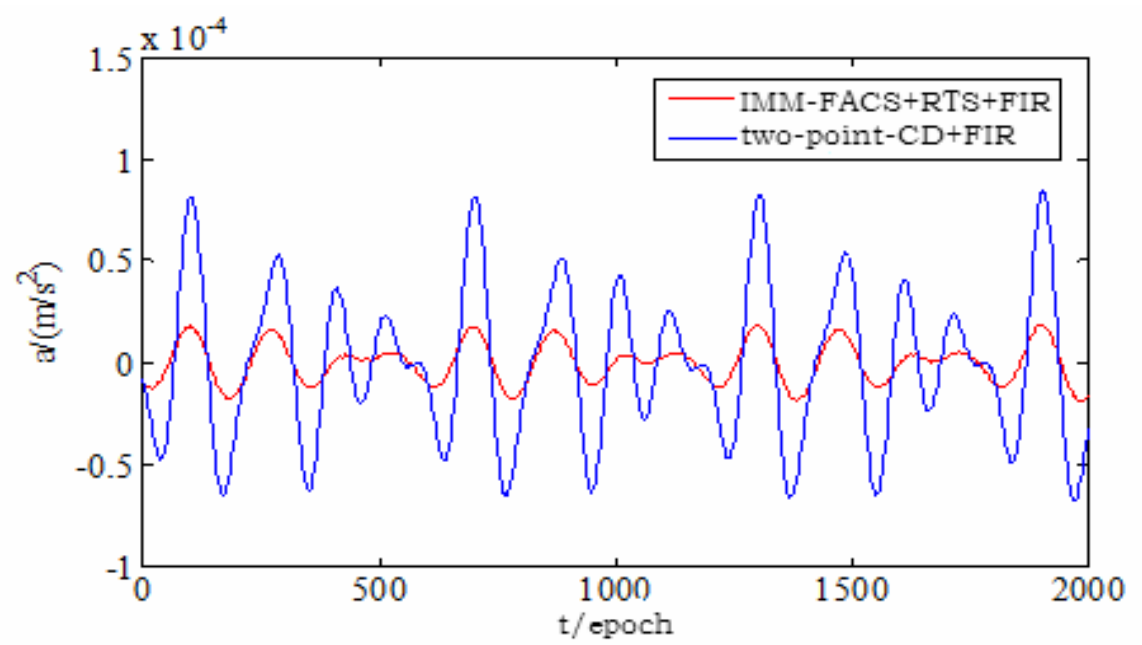

FIGURE III. THE DIFFERENCE BETWEEN THE TWO SOLUTIONS AND THE THEORETICAL VALUE

Table I shows the statistical results of the difference between the theoretical value and the results of carrier acceleration using the option 1 and 2.

TABLE I THE DIFFERENCE BETWEEN THE RESULTS OF OPTION 1AND 2 AND THE THEORETICAL VALUE

\begin{tabular}{|c|c|c|c|}
\hline & $\begin{array}{c}\text { before filtering } \\
\left(\mathrm{m} / \mathrm{s}^{2}\right)\end{array}$ & $\begin{array}{c}\text { after } \\
\text { filtering }\left(\mathrm{m} / \mathrm{s}^{2}\right)\end{array}$ & $\begin{array}{c}\text { compare } \\
\text { points }\end{array}$ \\
\hline Option1 & $2.511 \times 10^{-1}$ & $3.7555 \times 10^{-5}$ & 2000 \\
\hline Option2 & $1.7 \times 10^{-3}$ & $0.9757 \times 10^{-5}$ & 2000 \\
\hline
\end{tabular}

It can be seen from Figure 3 and Table I that the carrier acceleration accuracy of the IMM-FACS RTS solution is still higher than the position differential method after FIR low -pass filtering.

The simulation results show that the precision of carrier acceleration is higher than that of position differential method by using the method proposed in this paper, which improves the accuracy of carrier acceleration calculation and has an important application value for airborne gravimetry.

\section{CONCLUSIONS}

In this paper, the IMM-FACS RTS method is applied to the acceleration calculation in airborne gravimetry. For the characteristics of the "Current" Statistical Model (CS) acceleration parameters, the fuzzy membership function is used to fuzzyly correct the extreme value of acceleration, and the Interacting Multiple Model (IMM) algorithm is introduced into the filter frame of CS model, and finally the filtering results are improved by RTS smoothing. The simulation results show that the accuracy of the carrier acceleration by using this method is better than that of the position differential method and it can be applied in airborne gravimetry.

\section{ACKNOWLEDGMENTS}

The authore would like to acknowledge my professor for his valuable comments. And the study is supported by National Natural Science Foundation of China(61304001).

\section{REFERENCES}

[1] Zheng Wai, Zhang Guibin. Application of Adaptive Kalman Filter in Airborne Gravity Abnormal Solution[J]. Journal of Geophysics, 2016, 59(4): 1275-1283.

[2] Wang Jingbo, Xiong Shengqing, Guo Zhihong. Estimation of Vertical Carrier Acceleration in Airborne Gravimetry Using Kalman Smoothing Technique[J]. Journal of Geophysics, 2010, 25(3): 968-974.

[3] Li Xiaobing. GPS Positioning, Speed and Acceleration Resolution Technology and Application in Airborne Gravimetry[D]. Beijing: China University of Geosciences, 2010.

[4] Sun Zhongmiao, Xia Zheren. Design of FIR Low-pass Differential and Its Application in Airborne Gravimetry[J]. Journal of Geophysics, 2000, 43(6): 850-855.

[5] Bruton A M, Glennie C L, Schwarz K P. Differentiation for High Precision GPS Velocity and Acceleration Determination[J].GPS Solution, 1999, 2(4): 7-21.

[6 Xiao Yun, Xia Zheren. Determination of Carrier Motion Acceleration in Airborne Gravimetry[J]. Journal of Geophysics, 2003, 43(1): 62-66.

[7 Zhou Hongren, Jing Zhongliang, Wang Peide. Maneuvering Target Tracking[M]. Beijing: National Defense Industry Press, 1991: 134-144.

[8 Wang J H, Cao J, Wu D, et al. An Object Tracking Algorithm Based on the "Current" Statistical Model and the Multi-Feature Fusion[J]. Journal of Software. 2012, 9(7): 2000-2008.

[9 Liu Jianshu, Li Renhou, Liu Yunlong. An Interactive Multi-model Algorithm Based on "Current" Statistical Model[J]. Systems Engineering and Electronics, 2008, 30(7): 1351-1354. 